\title{
A Rare Cause of Ascites in a Young Woman Mimicking Peritoneal Carcinomatosis
}

\author{
Reza Fatemi ${ }^{1}$, Pardis Ketabi Moghadam ${ }^{2, *}$, Forough Mangeli ${ }^{3}$
}

1. Professor of gastroenterology and hepatology, Department of gastroenterology and hepatology, Taleghani hospital, Shahid Beheshti University of medical sciences, Tehran, Iran

2. Fellowship of gastroenterology and hepatology, Research Institute for Gastroenterology and Liver Diseases, Shahid Beheshti University of Medical Sciences, Tehran, Iran

3. Professor of pathology, Department of pathology, Taleghani hospital, Shahid Beheshti University of medical sciences, Tehran, Iran

\section{* Corresponding Author:}

Pardis Ketabi Moghadam, MD Research Institute for Gastroenterology and Liver Diseases of Shahid Beheshti University of Medical Sciences, Educational Taleghani Hospital, Tabnak St. Velenjak Region, Chamran High Way, Tehran, Iran Tel: + 982122432560 Fax: + $98212243-2570$

Email: Ketabimoghadam.p@gmail.com

Received: 28 Oct. 2020

Accepted: 10 Jun. 2021

\section{ABSTRACT}

Peritoneal granulomatous reaction to foreign body-like materials of a dermoid cyst is a rare condition and has always been challenging in diagnosis for physicians. Macroscopic view of granulomatous peritoneum, which mimics peritoneal carcinomatosis or peritoneal seeding originating from ovarian carcinoma, necessitates a detailed microscopic evaluation of peritoneum, which shows multinucleated giant cells and granuloma formation indicating a foreign body reaction in the peritoneum. To avoid unnecessary radical surgeries and salvage chemotherapies in these patients, a detailed microscopic evaluation of their peritoneum is necessary. The present study introduces a 23-year-old woman with new-onset ascites and an elevation of CA125 level suspicious of ovarian carcinoma but with a peritoneal microscopic finding pathognomonic of foreign body reaction.

\section{KEYWORDS:}

Ascites; Peritoneal carcinomatosis; Peritoneal granulomatous reaction

\section{Please cite this paper as:}

Fatemi R, Ketabi Moghadam P, Mangeli F. A Rare Cause of Ascites in a Young Woman Mimicking Peritoneal Carcinomatosis. Middle East J Dig Dis 2021;13:264-267. doi: 10.34172/mejdd.2021.234.

\section{INTRODUCTION}

Different reasons have been introduced for granulomatous inflammation of the peritoneum. Of which, bacterial and fungal infections and helminthiasis are more prevalent, and foreign body reaction caused by previous abdominopelvic manipulations lies in the next place after infectious agents. ${ }^{1}$ Neoplastic origins of granulomatous peritoneum are rare and are seen in teratomas or carcinoid tumors of the ovary and uterus. ${ }^{2}$

\section{CASE REPORT}

A 23-year-old woman presented to the Emergency Department of Taleghani Hospital, a teaching referral hospital in Tehran, Iran, with new-onset ascites gradually evolving since 1 month before her admission. She did not mention any change in bowel habit or any other gastrointestinalrelated complaints except mild nausea after ingestion, early satiety, and mild abdominal pain in particular in the periumbilical region along with ascites formation. She did not find to have weight loss, fever, neurological deficits indicating encephalopathy, irregular intervals of 


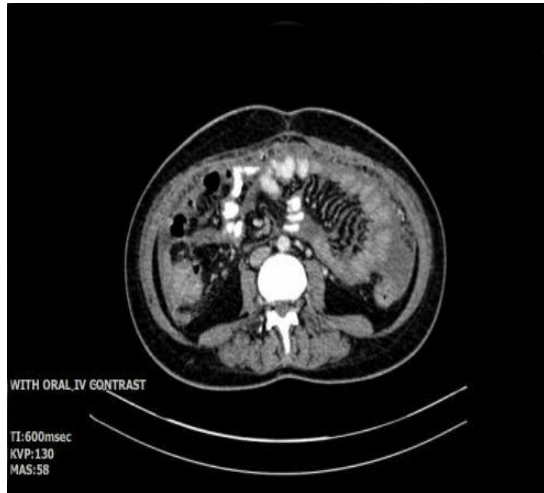

Fig. 1: Abdominopelvic CT reveals diffuse omental haziness

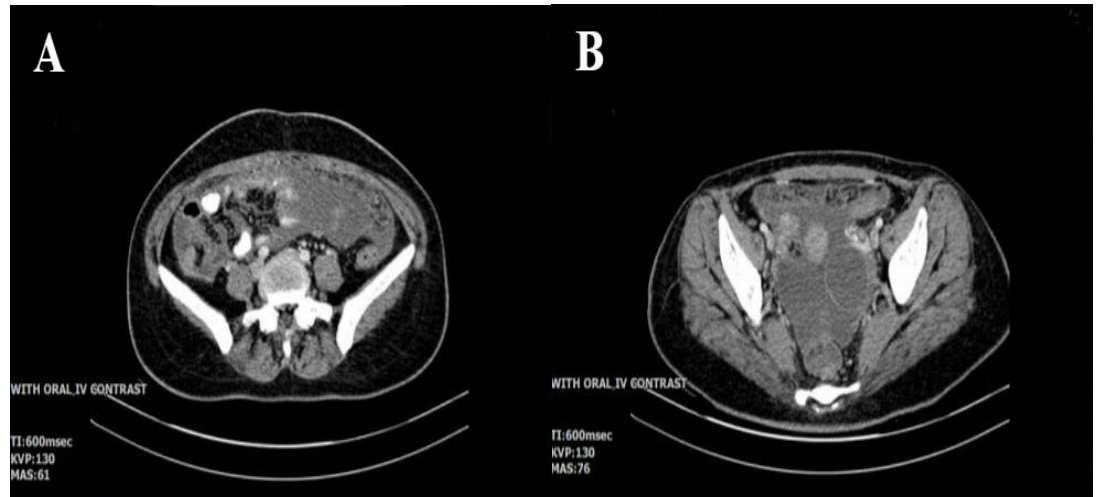

Fig. 2: The abdominopelvic CT shows an echogenic lesion $23 \times 40 \mathrm{~mm}$ in the right ovary consistent with a complicated dermoid cyst and a hemorrhagic follicle in the left ovary menstruation, hirsutism, deepening of the voice, acne, other manifestations of virilization, or other systemic and local signs and symptoms related to ascites-producing diseases. She denied smoking and consumption of alcohol or any other illicit drugs. Drug history was only notable for oral contraceptives. She was a housewife residing in Tehran and married, with a 2-year-old daughter. Her medical and surgical histories were only notable for cesarean section 2 years earlier and an axillary lipoma excision 10 years past. On admission, her physical exam was otherwise normal except for an abdominal distention with positive shifting dullness indicating severe ascites and mild bibasilar crackles on auscultation along with dullness to percussion in the lung bases. Vital signs were stable. We decided to proceed with complete examinations for ascites. Abdominopelvic ultrasonography revealed an echogenic lesion $23 \times 40 \mathrm{~mm}$ in the right ovary consistent with a complicated dermoid cyst (further evaluations by transvaginal sonography proved the same results) and a hemorrhagic follicle in the left ovary. Abdominal vessels were unremarkable on the color doppler study. An abdominopelvic computed tomography (CT) was done, which is seen in figures 1 , and 2. A diagnostic and therapeutic ascites fluid paracentesis was done. The analysis demonstrated a high SAAG (Serum Ascites Albumin Gradient) and low protein nature with near-normal cell count and cell differentiation without any malignant cells in cytology. Above that, bacterial, fungal, and mycobacterial staining and culture of ascitic fluid were totally negative.
Endoscopic and colonoscopic findings were normal. Echocardiography was unremarkable. Blood chemistry tests consisted of liver function and kidney function tests, complete blood count, hormonal tests such as ovarian, adrenal, and thyroid function tests, serum total protein and albumin, serum protein electrophoresis, tumor markers including AFP (Alpha Fetoprotein) and CEA (Carcinoembryonic Antigen) except for an elevation in CA-125 (121 U/L with normal range of $35 \mathrm{U} / \mathrm{L})$. Rheumatologic tests, including ANA and anti ds-DNA, quantiferron test, urinalysis, and 24-hour-urine protein evaluation, were all normal. A magnetic resonance imaging (MRI) and transvaginal ultrasonography (TVS) for more evaluation of mentioned complicated ovarian cyst were requested, which revealed few paraaortic lymph nodes, bilateral adnexal diffusion restriction spots (probably metastatic nodules), a thin wall left and right ovarian cysts, moderate free fluid in the pelvic cavity, diffuse omental haziness, and peritoneal nodularity suspicious of small metastatic ovarian nodules. Given the MRI report suggestive of ovarian cancer and an elevated serum CA125, she underwent a diagnostic laparoscopic surgery for a peritoneal biopsy sampling. Findings revealed a peritoneal nodularity suspicious of a malignant process. The pathology of the peritoneal specimen is depicted in figure 3. Microscopic evaluation of peritoneum was consistent with fibroadipose tissue with chronic non-necrotizing granulomatous inflammation. Multinucleated giant cells containing calcified material and foreign body crystalloids 


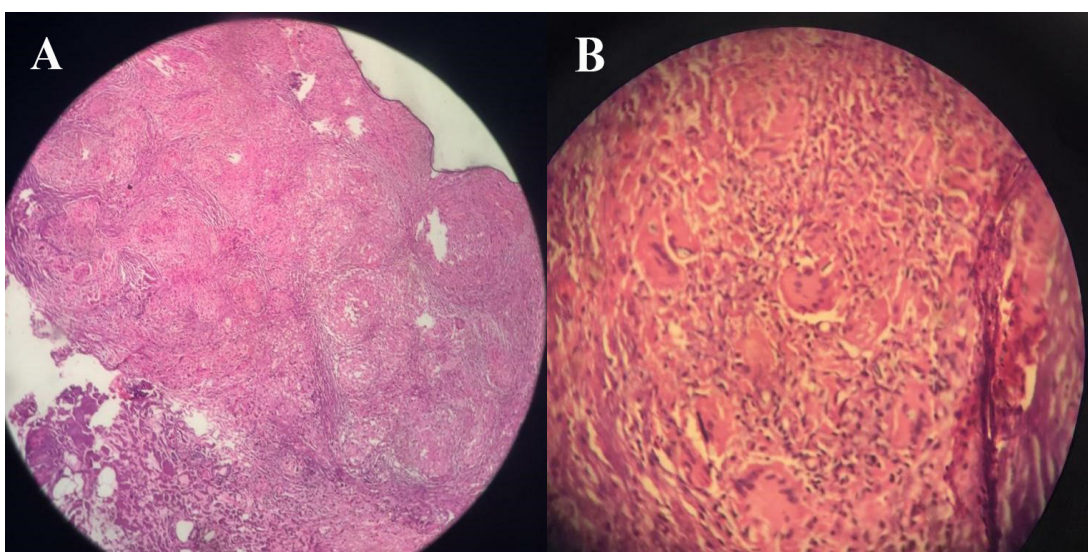

Fig. 3: The peritoneal specimen reveals fibroadipose tissue with chronic non-necrotizing granulomatous inflammation. Multinucleated giant cells containing calcified material and foreign body crystalloids are also seen

were detected. No evidence of fungal elements, acid-fast bacilli, or malignancy was detected in the specimen. Further evaluations for mycobacterium tuberculosis by real-time PCR method were also negative. Because of a complicated cystic lesion in the left ovary besides an elevated CA125 level, the patient was asked to have an ultrasound-guided biopsy sampling from the omentum to definitely rule out a peritoneal seeding originating from ovarian cancer. The second biopsy was again compatible with dermoid cyst resulting in an extensive foreign body-type granulomatous inflammation in the peritoneum without any evidence of malignancy. The giant cell indicating a foreign body reaction is depicted in figure 4. In a 1-year follow-up, the patient's ascites was completely resolved, and she did not have any abdominal pain, weight loss, abnormal uterine bleeding, or any other alarming signs indicating further followup.

\section{DISCUSSION}

Peritoneal granulomatous foreign body reaction is a rare tumor-like lesion with infectious or non-infectious etiology. ${ }^{3}$ Non-infectious types are usually secondary to neoplasms of the female genital tract like endometrium and cervix carcinoma and rarely polypoid adenomyomas of the uterus. ${ }^{2}$ peritoneal granuloma consists of laminated keratin deposits surrounded by foreign body giant cells and fibrous tissue, as we can see in figure 3 . The differential diagnosis includes peritoneal granulomas in response to keratin derived from other sources, including amniotic

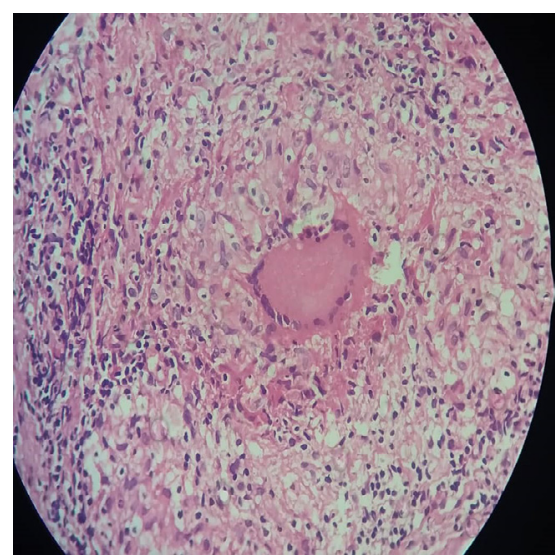

Fig. 4: A giant multinucleated cell in omental specimen

fluid, manipulation of omentum during surgeries such as cesarean section, and ovarian dermoid cysts. ${ }^{3}$ So, an expert pathologist should examine the specimen to exclude the presence of tumoral cells in the sample. It is recommended that radical surgery or other antineoplastic therapies be commenced only with a definite microscopic diagnosis of cancer. ${ }^{4}$ Given the abdominopelvic ultrasonography, the presence of a complicated cystic lesion in the ovary suspicious of malignancy made us proceed with a more accurate diagnostic tool to rule out a peritoneal seeding originating from the ovary. Overall, TVS as an excellent imaging modality for the evaluation of ovarian pathology is as accurate as MRI in the identification and accurate characterization of ovarian dermoid. ${ }^{5}$ We evaluated our patient by TVS and MRI. The results were both compatible with the dermoid cyst. Rupture into the peritoneum is a known serious complication of dermoid cysts, which may cause a remarkable granulomatous reaction leading to the formation of reactive nodules mimicking generalized carcinomatosis or tuberculous peritonitis. ${ }^{6,78} \mathrm{~A}$ sudden rupture may lead to acute peritonitis, but a chronically leaking cyst may induce granulomatous peritonitis characterized by multiple small white peritoneal implants and dense adhesions as was reported in our patient. ${ }^{6,7}$ Above that, there was an omental manipulation due to cesarean section as another reason for peritoneal granulomatous foreign body reaction in the medical history of our patient. Once the diagnosis of granulomatous peritonealis is established, the underlying cause is suggested to be treated. however, spontaneous recovery is seen in most cases, and only 
patients with significant inflammatory masses may benefit from the use of corticosteroids. ${ }^{9}$ A 1-year follow-up of our patient revealed spontaneous gradual subsidence of peritoneal lesions and ascites without any treatment.

\section{ETHICAL APPROVAL}

There is nothing to be declared.

\section{CONFLICT OF INTEREST}

The authors declare no conflict of interest related to this work.

\section{REFERENCES}

1. Clement PB. Reactive tumor-like lesions of the peritoneum. Am J Clin Pathol 1995;103: 673-5. doi: 10.1093/ajcp/103.6.673.

2. Kim KR, Scully RE. Peritoneal keratin grarmlomas with carcinomas of endometrium and atypical polypoid adenomyoma endometrium. Am J Surg Pathol 1990;14:92532. doi: 10.1097/00000478-199010000-00005.

3. Clement PB: Tumors of the peritonium; in Fletcher DMC (ed): Diagnostic Histopathology of Tumors. 3rd ed. London: Churchil Livingstone 2007:881-884.

4. Wiberg N, Kiss K, Dalsgaard L. Lipogranuloma peritonealis caused by spontaneous rupture of a benign cystic ovarian teratoma. Acta Obstet Gynecol Scand 2003:82:91-4. doi: 10.1034/j.16000412.2003.820117.x.

5. Jain KA, Friedman DL, Pettinger TW, Alagappan R, Brooke JR, Sommer FG. Adnexal masses: Comparison of specificity of endovaginal US and pelvic MR imaging. Radiology 1993;186:697-704. doi: 10.1148/ radiology.186.3.8430177.

6. Quer EA, Dockerty MB, Mayo CW. Ruptured dermoid cyst of the ovary simulating abdominal carcinomatosis. Report of a case. Proc Staff Meet Mayo Clinic 1951;26: 489-98.

7. Stem JL, Buscema J, Rosenshein NB, Woodruff JD. Spontaneous rupture of benign cystic teratoms. Obstet Gynecol 1981;57: 363-6.

8. Ranney B. Iatrogenic spillage from benign cystic teratoma causing severe peritoneal granulomas and adhesions. Report of a case. Obstet Gynecol 1970;35: 562-4.

9. Stuart GCE, Smith JP. Ruptured benign cystic teratomas mimicking gynecologic malignancy. Gynecol Oncol 1983;16:139-43. doi: 10.1016/0090-8258(83)900197. 